\title{
Molecular Breeding of Rice Restorer Lines and Hybrids for Brown Planthopper (BPH) Resistance Using the Bph14 and Bph15 Genes
}

\author{
Hongbo Wang, Shengtuo Ye and Tongmin Mou*
}

\begin{abstract}
Background: The development of hybrid rice is a practical approach for increasing rice production. However, the brown planthopper (BPH), Nilaparvata lugens Stål, causes severe yield loss of rice (Oryza sativa L.) and can threaten food security. Therefore, breeding hybrid rice resistant to BPH is the most effective and economical strategy to maintain high and stable production. Fortunately, numerous BPH resistance genes have been identified, and abundant linkage markers are available for molecular marker-assisted selection (MAS) in breeding programs. Hence, we pyramided two BPH resistance genes, Bph14 and Bph15, into a susceptive CMS restorer line Huahui938 and its derived hybrids using MAS to improve the BPH resistance of hybrid rice.

Results: Three near-isogenic lines (NILs) with pyramided Bph14 and Bph15 were obtained by molecular marker-assisted backcross (MAB) and phenotypic selection. The genomic components of these NILs were detected using the whole-genome SNP (Single nucleotide polymorphism) array, RICE6K, suggesting that the recurrent parent genome (RPG) recovery of the NILs was $87.88,87.70$ and $86.62 \%$, respectively. BPH bioassays showed that the improved NILs and their derived hybrids carrying homozygous Bph14 and Bph15 were resistant to $\mathrm{BPH}$. However, the hybrids with heterozygous Bph14 and Bph15 remained susceptible to $\mathrm{BPH}$. The developed NILs showed no significant differences in major agronomic traits and rice qualities compared with the recurrent parent. Moreover, the improved hybrids derived from the NILs exhibited better agronomic performance and rice quality compared with the controls under natural field conditions.
\end{abstract}

Conclusions: This study demonstrates that it is essential to stack Bph14 and Bph15 into both the maternal and paternal parents for developing BPH-resistant hybrid rice varieties. The SNP array with abundant DNA markers is an efficient tool for analyzing the RPG recovery of progenies and can be used to monitor the donor segments in NILs, thus being extremely important for rice molecular breeding.

Keywords: Rice, Brown Planthopper (BPH), Bph14 and Bph15, MAS, Hybrid Rice

\section{Background}

Rice (Oryza Sativa L.) is one of the most important food sources for more than half of the world population. It is predicted that an additional 116 million tons of rice will be needed by 2035 to feed the growing population (Seck et al. 2012). However, the brown planthopper

\footnotetext{
* Correspondence: tongmin58@mail.hzau.edu.cn

National Key Laboratory of Crop Genetic Improvement and National Center of Plant Gene Research (Wuhan), Huazhong Agricultural University, Wuhan 430070, China
}

(BPH, Nilaparvata lugens Stål), which sucks the phloem sap of the rice leaf sheath and transmits viral diseases such as rice grassy stunt virus (RGSV), rice ragged stunt virus (RRSV) and rice wilted stunt virus (RWSV), often leads to severe yield losses in the agricultural industry (Fujita et al. 2013).

More than $90 \%$ of the world's rice is grown in Asian countries. China is the largest rice-producing country in the world, although the planting area is less than that in India. The total rice production in 2015 was about 
208 million tons in China, which accounted for more than $20 \%$ of the world's total rice yield. Widely grown hybrid rice is one of the main factors contributing to the increase in rice yield in China since the 1970s. The IRRI estimates that planthoppers cause annual yield losses of 1 to 2 million tons of paddy rice in China (Fujita et al. 2013). The total planting area of rice infested by BPH in China exceeded 25 million hectares between 2005 and 2007 (Qiu et al. 2012). Rapid expansion of the planting area of hybrid rice is probably one of the main causes of the $\mathrm{BPH}$ crisis because most of the hybrid rice varieties released in China are susceptible to BPH (Hu et al. 2012). The area planted to hybrid rice has already increased by over $50 \%$ in China, and the planted area of hybrid rice in India and other Asian countries such as Vietnam, Myanmar and Indonesia, has also increased in recent years (Horgan and Crisol 2013). Therefore, it is a very urgent task to develop hybrid rice cultivars with $\mathrm{BPH}$ resistance as soon as possible.

Fujita et al. (2013) reviewed the progress in the identification of $\mathrm{BPH}$ resistance genes. To date, 29 major $\mathrm{BPH}$ resistance genes/loci have been identified from cultivated, landrace and wild species of Oryza (Fujita et al. 2013; Wang et al. 2015). These are located on 8 of the 12 chromosomes in rice, whereas no $\mathrm{BPH}$ genes have been identified on chromosomes 1, 5, 7 and 8. Multiple BPHresistance loci are closely positioned or overlap on the same chromosome regions, and four gene clusters have been designated as A, B, C and D (Fujita et al. 2013). The continuing validation of $\mathrm{BPH}$-resistance loci offers possibilities for rice molecular breeding.

Compared with traditional chemical control, a host plant resistance breeding strategy is a more effective and environmentally friendly method to control $\mathrm{BPH}$ damage (Jairin et al. 2010; Sun et al. 2005). Molecular markerassisted selection (MAS) is a highly efficient approach for plant-breeding scientists to select target genes both rapidly and precisely (Tanksley et al. 1989). It has been certified that pyramiding of resistance genes can provide stronger and more durable resistance for susceptible rice cultivars in contrast to single gene introgression. Myint et al. (2012) found that the BPH resistance level of Bph $25+$ Bph26-NILs was significantly higher than Bph25NILs or Bph26-NILs. Qiu et al. (2012) found that the indica rice 9311 and japonica rice Nipponbare lines with pyramided Bph6 and Bph12 had obviously less damage compared to monogenic lines.

Bph14 and Bph15, separately located on chromosome 3 and 4 in B5 (a breeding line derived from O. officinalis), are two widely adopted genes in the BPH-resistance breeding practice (Huang et al. 2001; Li et al. 2006; Xia et al. 2010; Zhu et al. 2013a; Zhu et al. 2013b; $\mathrm{Hu}$ et al. 2012; $\mathrm{Hu}$ et al. 2015; Cai et al. 2015). Bph14 was the first cloned $\mathrm{BPH}$ resistance gene and encodes a coiled-coil, nucleotide-binding and leucine-rich repeat (CC-NB-LRR) protein (Du et al. 2009). Bph15 was previously mapped to a $47-\mathrm{kb}$ region, but the latest research showed that the region containing Bph15 was nearly $580 \mathrm{~kb}$ between DNA marker g12140-2 and marker T12 in B5, which originated from the C genome of O. officinalis (Yang et al. 2004; Lv et al. 2014). A previous study found that the BPH-resistance effect of Bph15 was higher than that of Bph14. Moreover, both Bph14 and Bph15 exhibit partial dominance and have a pronounced dosage effect on the resistance to $\mathrm{BPH}$ in hybrids (Li et al. 2011; Hu et al. 2012).

Huahui938 is an elite rice restorer line of CMS selected by our laboratory in previous years. We developed several hybrids with high yield, good quality and resistance to blast using Huahui938 as a male parent. However, Huahui938 and its derived hybrids are susceptible to $\mathrm{BPH}$. In the present study, we further improved the BPH-resistance of Huahui938 and its derived hybrids by pyramiding Bph14 and Bph15 using marker-assisted backcross selection (MAB) and whole-genome SNP array analysis, following the effective evaluation of pyramided genes conferring resistance to $\mathrm{BPH}$ in the improved NILs and the corresponding hybrids.

\section{Results \\ Development of NILs with Bph14 and Bph15 Genes Using $M A B$}

$\mathrm{F}_{1}$ progenies were obtained from the cross between Huahui938 (recurrent parent) and B5 (donor parent of Bph14 and Bph15). Based on PCR analysis, true $\mathrm{F}_{1}$ plants containing Bph14 and Bph15 were selected and backcrossed with Huahui938 to produce $\mathrm{BC}_{1} \mathrm{~F}_{1}$. Among 30 $\mathrm{BC}_{1} \mathrm{~F}_{1}$ plants, five containing heterozygous Bph14 and $B p h 15$ loci were used for further backcrossing to produce $\mathrm{BC}_{2} \mathrm{~F}_{1}$. Among $100 \mathrm{BC}_{2} \mathrm{~F}_{1}$ plants, two individuals containing Bph14 and Bph15 and with a phenotype similar to Huahui938 were used for subsequent backcrossing. Among $60 \mathrm{BC}_{3} \mathrm{~F}_{1}$ plants, 19 were identified as containing Bph14 and Bph15, and two plants (designed as HB13002-5 and HB13002-50) with identical agronomic performance to Huahui938 were self-crossed to produce $\mathrm{BC}_{3} \mathrm{~F}_{2}$ seeds. Then, in two $\mathrm{BC}_{3} \mathrm{~F}_{2}$ populations of 100 plants, eight plants containing homozygous Bph14 and Bph15 were selected to produce $\mathrm{BC}_{3} \mathrm{~F}_{3}$ (Fig. 1a). Next, eight $\mathrm{BC}_{3} \mathrm{~F}_{3}$ family lines were obtained and further confirmed by PCR analysis of the target loci, which suggested that both Bph14 and Bph15 were homozygous in all family lines (Fig. 1b). Finally, three lines with no phenotype segregation of agronomic performance, including number of days to heading, plant height, tiller number, plant type and panicle type, among individuals of the population were designed as HB13002-5-30-10, HB13002-5-31-24 and HB13002-50-9-7. 
a

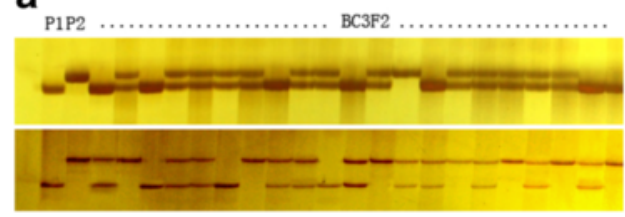

b

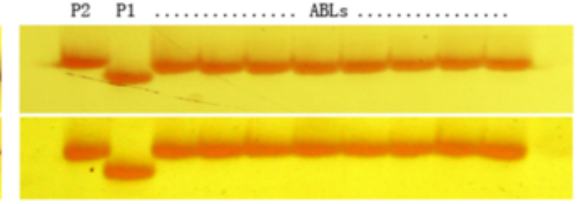

Fig. $1 P C R$ analysis of $B C_{3} F_{2}$ plants from the cross between Huahuig38 and $B 5$ (a) and resistance gene confirmation of $A B L s$ in $B C_{3} F_{3}(\mathbf{b})$. a Upper panel: PCR analysis of Bph14 with marker 76-2; Lower panel: PCR analysis of Bph15 with marker MS5; b Upper panel: Bph14 gene confirmation with marker 76-2; Lower panel: Bph15 gene confirmation with marker InD4. P : Huahui938; P2: B5; ABLs: advanced backcrossed breeding lines

The genetic backgrounds of the three selected nearisogenic lines (NILs) were assayed using the wholegenome SNP array RICE6K. A total of 550 SNP markers showed polymorphism between Huahui938 and B5. The recurrent parent genome (RPG) recoveries of HB130025-30-10, HB13002-5-31-24 and HB13002-50-9-7 were $87.88,87.70$ and $86.62 \%$, respectively. The haplotype maps also implied that the segments containing target loci were successfully transferred from the donor parent B5 into the selected NILs, and no heterozygous loci remained at the whole-genome scale of the three improved lines (Fig. 2). These results suggested that positive selection of target genes and continuous phenotypic selection can be used to achieve the genetic background of the recurrent parent with a recovery rate higher than $80 \%$ and can also be used to rapidly establish the whole-genome homozygosity of the selected lines.

\section{Resistance Reaction of the Selected NILs and Hybrids in Response to $\mathrm{BPH}$}

The resistance levels to BPH of the selected NILs and their derived hybrids, along with the controls, were evaluated at the seedling stage. The evaluation results showed that B5 and the CMS line Luohong4A with two homozygous resistance genes were resistant against $\mathrm{BPH}$, with scores of 3.0 and 2.3 , respectively. The three selected NILs, HB13002-5-30-10, HB13002-5-31-24 and HB13002-50-9-7, were also resistant against BPH, similar to the resistance controls, with scores of 2.3, 2.3 and 3.0, respectively. Huahui938, TN1, Quan9311A, Fengliangyou4 and Quan9311A/Huahui938, without resistance genes, were susceptible or highly susceptible to $\mathrm{BPH}$, and their scores ranged from 7.7 to 9.0. Interestingly, the hybrids with homozygous Bph14 and Bph15 genes, i.e., Luohong4A/B5, Luohong4A/HB13002-5-30-10, Luohong4A/HB13002-5-31-24 and Luohong4A/HB1300250-9-7, were resistant against $\mathrm{BPH}$, and their $\mathrm{BPH}$ resistance scores were 2.3-3.0. However, the hybrids containing heterozygous Bph14 and Bph15 genes, i.e., Luohong4A/ Huahui938, Quan9311A/B5, Quan9311A/HB13002-530-10, Quan9311A/HB13002-5-31-24 and Quan9311A/ HB13002-50-9-7, were susceptible or highly susceptible to $\mathrm{BPH}$, similar to the materials without $\mathrm{BPH}$-resistant genes (Fig. $3 \mathrm{a}$ and $\mathrm{b}$ ). Taken together, these results indicate
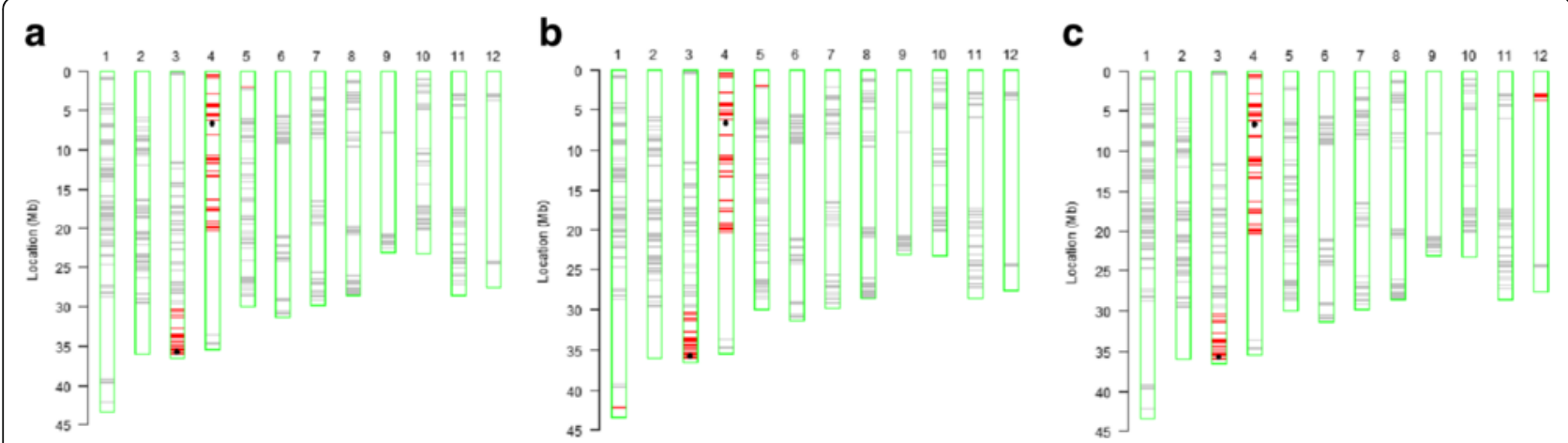

Fig. 2 Genetic background assay of the three selected NILs detected using the RICE6K array. a HB13002-5-30-10, b HB13002-5-31-24 and c HB13002-50-9-7. The black dots indicate the positions of two target genes, Bph14 on chromosome 3 and Bph15 on chromosome 4. The red lines indicate the SNP loci with homozygous genotypes where genomic fragments of the donor parent B5 were introgressed, the gray lines indicate the SNP loci with the same genotypes as the recurrent parent Huahui938, and the white boxes indicate non-polymorphism fragments between recurrent and donor parents 


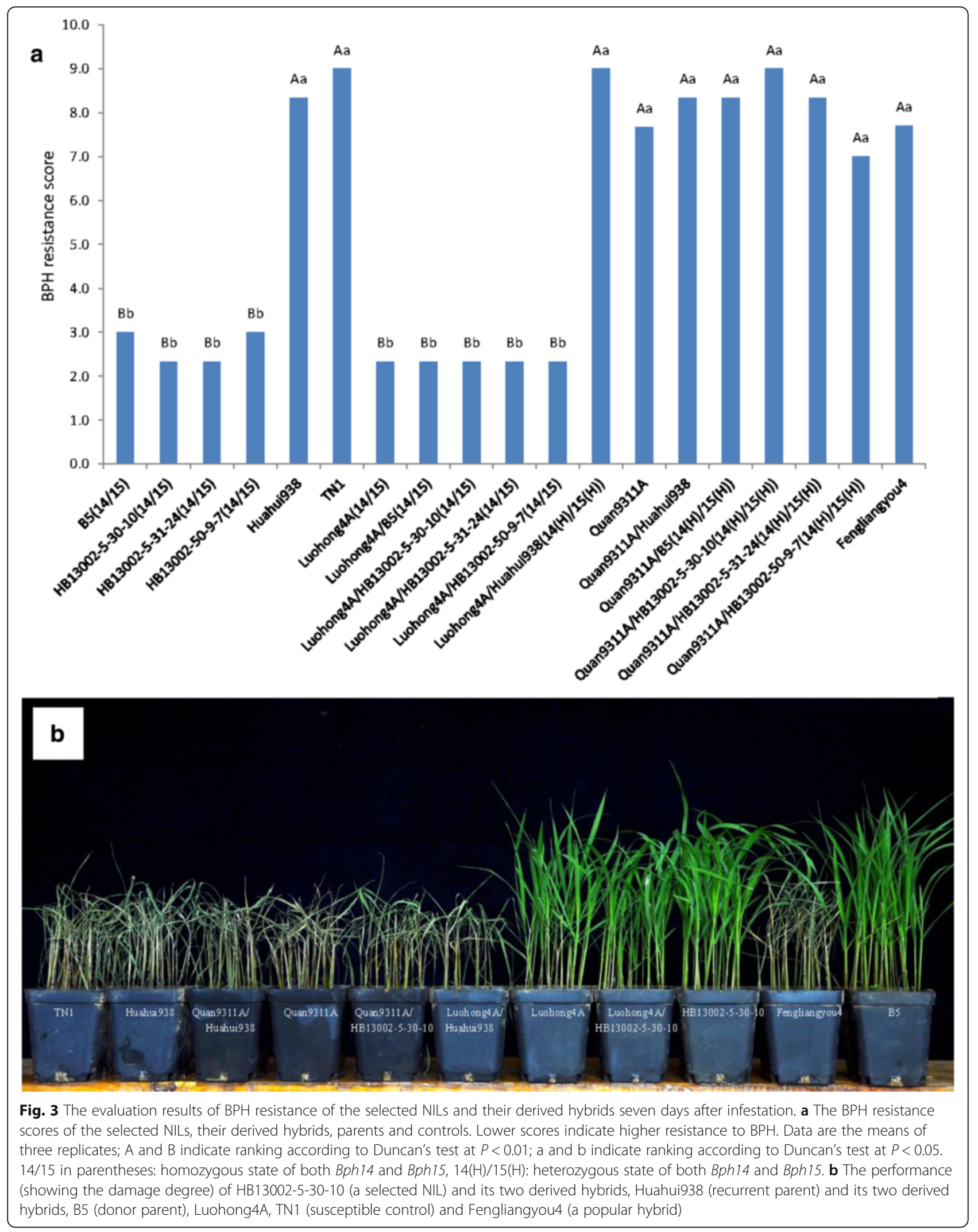


that the protection of hybrid rice from damage caused by $\mathrm{BPH}$ requires the simultaneous genetic improvement of maternal and paternal parents with the use of Bph14 and Bph15.

\section{The Performance of Agronomic Traits of the NILs and Their Hybrids}

To test whether the other phenotypic traits of the selected NILs and their derived hybrids were identical to those of the recurrent parent and its derived hybrids under normal conditions, we examined eight agronomic traits and six rice quality traits of the selected NILs and hybrids planted at Wuhan without BPH damage (Table 1). Traits such as panicle length (PL), panicle number per plant $(\mathrm{PN})$, number of grains per panicle (NGP), fertility of the spikelet (FER), 1000-grain weight $(\mathrm{GW})$, grain yield per plant (GY), chalkiness degree (CD), amylose content (AC) and alkali spreading value (ASV) of the three selected NILs showed no significant difference compared with Huahui938. However, the number of days to heading (DTH) of HB13002-50-9-7 was 3 days longer than that of Huahui938, the PN of HB13002-50-9-7 was 2.8 lower than Huahui938 and the plant height (PH) of HB13002-30-10 was approximately $5.3 \mathrm{~cm}$ higher than that of Huahui938. Moreover, the head rice rate (HR) of the three selected NILs was higher than that of Huahui938, which is a desirable quality trait for rice improvement.

The evaluation results of the agronomic and rice quality traits of hybrids derived from the selected NILs showed that most of traits were identical to those of the hybrids derived from the recurrent parent, Huahui938 (Table 1). Among them, the GY of improved hybrid Luohong4A/HB13002-9-7 was not significantly different from that of the control Luohong4A/Huahui938. In addition, all three improved hybrids had better rice quality with a higher HR and significantly lower $\mathrm{CK}$ and $\mathrm{CD}$ compared with Luohong4A/Huahui938. Furthermore, most of the agronomic traits of the three improved Quan9311A/ NILs were identical to the control Quan9311A/Huahui938 except that Quan9311A/ HB13002-30-10 had a higher GW. Additionally, all three hybrids derived from Quan9311A had a better rice quality with a significantly lower chalky grain rate (CK). Compared to Fengliangyou4, a popular and outstanding hybrid variety in agricultural production, the hybrid Luohong4A/HB13002-9-7 showed similar GY but shorter DTH and lower PH. Meanwhile, the hybrid Quan9311A/HB13002-9-7 had a significantly higher GY and lower CK compared with Fengliangyou4 (Table 1). The BPH-resistant NILs and hybrids, which were identical to the recurrent parent and its derived hybrids with respect to the agronomic and rice quality traits, are more strongly desired.

Table 1 The agronomic and rice quality traits of the three selected NILs and their derived hybrids

\begin{tabular}{|c|c|c|c|c|c|c|c|c|c|c|c|c|c|c|}
\hline \multirow[t]{2}{*}{ Entire } & \multicolumn{8}{|c|}{ Agronomic traits } & \multicolumn{6}{|c|}{ Traits of grain qualities } \\
\hline & DTH (d) & $\mathrm{PH}(\mathrm{cm})$ & $\mathrm{PL}(\mathrm{cm})$ & PN & NGP & FER (\%) & GW (g) & GY (g) & $\mathrm{HR}(\%)$ & L/W & CK (\%) & CD (\%) & $A C(\%)$ & ASV \\
\hline luahui 938 & $92.0^{\mathrm{b}}$ & $117.5^{c}$ & $27.1^{b}$ & $14.4^{\mathrm{b}}$ & $137.2^{a}$ & $77.66^{a}$ & $24.6^{\mathrm{b}}$ & $29.2^{a}$ & $53.9^{c}$ & $2.87^{b}$ & $11.4^{\mathrm{bc}}$ & $4.0^{b}$ & $10.2^{b}$ & $6.0^{a}$ \\
\hline HB13002-5-30-10 & $91.7^{\mathrm{b}}$ & $122.8^{\mathrm{a}}$ & $28.1^{\mathrm{ab}}$ & $13.3^{b c}$ & $149.3^{\mathrm{a}}$ & $81.07^{\mathrm{a}}$ & $25.6^{\mathrm{b}}$ & $30.0^{\mathrm{a}}$ & $57.8^{\mathrm{b}}$ & $3.03^{\mathrm{a}}$ & $13.9^{\mathrm{b}}$ & $4.2^{\mathrm{b}}$ & $10.6^{\mathrm{ab}}$ & $6.0^{\mathrm{a}}$ \\
\hline B13002-5-31-24 & $92.0^{\mathrm{b}}$ & $118.6^{\mathrm{bc}}$ & $27.3^{\mathrm{ab}}$ & $13.4^{\mathrm{bc}}$ & $137.8^{\mathrm{a}}$ & $77.39^{\mathrm{a}}$ & $24.9^{b}$ & $29.8^{\mathrm{a}}$ & $59.0^{\mathrm{ab}}$ & $2.90^{\mathrm{b}}$ & $11.0^{\mathrm{bc}}$ & $3.1^{\mathrm{b}}$ & $10.2^{\mathrm{b}}$ & $5.9^{\mathrm{a}}$ \\
\hline B13002-50-9-7 & $95.0^{a}$ & $120.3^{\mathrm{bc}}$ & $28.3^{\mathrm{ab}}$ & $11.6^{\mathrm{d}}$ & $145.2^{\mathrm{a}}$ & $82.04^{\mathrm{a}}$ & $25.1^{\mathrm{b}}$ & $28.3^{\mathrm{a}}$ & $58.1^{\mathrm{b}}$ & $2.90^{\mathrm{b}}$ & $8.7^{c}$ & $3.5^{\mathrm{b}}$ & $10.7^{\mathrm{ab}}$ & $6.0^{\mathrm{a}}$ \\
\hline B5 & $86.7^{c}$ & $113.8^{d}$ & $28.8^{\mathrm{a}}$ & $16.5^{\mathrm{a}}$ & $115.7^{b}$ & $81.05^{\mathrm{a}}$ & $27.6^{\mathrm{a}}$ & $29.4^{\mathrm{a}}$ & $61.1^{\mathrm{a}}$ & $2.77^{c}$ & $34.0^{\mathrm{a}}$ & $10.5^{\mathrm{a}}$ & $11.1^{\mathrm{a}}$ & $2.0^{\mathrm{b}}$ \\
\hline Luohong4A/Huahui938 & $84.0^{\mathrm{b}}$ & $105.3^{\mathrm{bc}}$ & $25.1^{\mathrm{ab}}$ & $11.1^{\mathrm{b}}$ & $180.8^{b c}$ & $79.93^{\mathrm{a}}$ & $25.2^{\mathrm{bc}}$ & $37.2^{\mathrm{a}}$ & $61.4^{\mathrm{b}}$ & $2.87^{\mathrm{a}}$ & $53.7^{\mathrm{a}}$ & $21.2^{\mathrm{a}}$ & $20.9^{\mathrm{a}}$ & $3.4^{\mathrm{bc}}$ \\
\hline -uohong4A/HB13002-5-30-10 & $84.0^{\mathrm{b}}$ & $106.4^{\mathrm{b}}$ & $25.3^{\mathrm{ab}}$ & $11.4^{\mathrm{b}}$ & $171.7^{c}$ & $75.93^{\mathrm{a}}$ & $25.6^{\mathrm{b}}$ & $33.8^{\mathrm{b}}$ & $65.1^{\mathrm{a}}$ & $2.90^{\mathrm{a}}$ & $31.5^{c}$ & $11.0^{c}$ & $19.2^{b}$ & $3.8^{b}$ \\
\hline -uohong4A/HB13002-5 & $83.3^{b}$ & $103.0^{c}$ & $25.3^{\mathrm{ab}}$ & $11.5^{\mathrm{b}}$ & $176.2^{\mathrm{bc}}$ & $70.77^{\mathrm{bc}}$ & $25.6^{\mathrm{b}}$ & $33.7^{\mathrm{b}}$ & $65.1^{a}$ & $2.90^{\mathrm{a}}$ & $37.0^{\mathrm{bc}}$ & $12.3^{\mathrm{bc}}$ & $19.3^{b}$ & $3.1^{b c}$ \\
\hline Luohong4A/HB13002-50-9-7 & $84.7^{\mathrm{b}}$ & $106.5^{\mathrm{b}}$ & $25.5^{\mathrm{a}}$ & $11.3^{b}$ & $186.6^{\mathrm{b}}$ & $74.93^{\mathrm{ab}}$ & $24.7^{c}$ & $35.3^{\mathrm{ab}}$ & $65.8^{\mathrm{a}}$ & $2.90^{\mathrm{a}}$ & $29.4^{c}$ & $11.2^{\mathrm{bc}}$ & $20.9^{\mathrm{a}}$ & $3.7^{b c}$ \\
\hline Luohong4A/B5 & $80.1^{c}$ & $102.9^{c}$ & $24.7^{\mathrm{b}}$ & $13.0^{\mathrm{a}}$ & $154.0^{d}$ & $63.4^{c}$ & $26.9^{\mathrm{a}}$ & $27.0^{c}$ & $65.6^{\mathrm{a}}$ & $2.87^{\mathrm{a}}$ & $45.8^{\mathrm{ab}}$ & $13.8^{\mathrm{b}}$ & $19.1^{\mathrm{b}}$ & $2.7^{\mathrm{c}}$ \\
\hline Fengliangyou4(CK) & $88.0^{\mathrm{a}}$ & $117.3^{\mathrm{a}}$ & $24.9^{\mathrm{ab}}$ & $9.6^{c}$ & $203.7^{\mathrm{a}}$ & $81.62^{a}$ & $27.46^{\mathrm{a}}$ & $35.2^{\mathrm{b}}$ & $64.5^{\mathrm{a}}$ & $2.77^{b}$ & $29.2^{c}$ & $8.3^{d}$ & $14.9^{c}$ & $5.9^{\mathrm{a}}$ \\
\hline Luan9311A/Huahui938 & $88.7^{a}$ & $115.2^{\mathrm{a}}$ & $27.3^{b}$ & $12.0^{\mathrm{a}}$ & $181.1^{b c}$ & $82.89^{a b}$ & $27.5^{\mathrm{b}}$ & $38.6^{\mathrm{ab}}$ & $64.3^{a}$ & $2.77^{\mathrm{a}}$ & $18.0^{c}$ & $6.1^{b}$ & $13.2^{b}$ & $5.8^{\mathrm{a}}$ \\
\hline Quan9311A/HB13002-5-30-10 & $88.7^{\mathrm{a}}$ & $115.0^{\mathrm{a}}$ & $27.9^{\mathrm{ab}}$ & $11.6^{\mathrm{a}}$ & $179.5^{\mathrm{bc}}$ & $86.11^{\mathrm{a}}$ & $28.4^{\mathrm{a}}$ & $39.7^{\mathrm{ab}}$ & $64.6^{\mathrm{a}}$ & $2.73^{a}$ & $17.2^{c}$ & $6.0^{\mathrm{bc}}$ & $13.3^{b}$ & $6.2^{\mathrm{a}}$ \\
\hline Quan9311A/HB13002-5-31-24 & $88.7^{\mathrm{a}}$ & $114.9^{\mathrm{a}}$ & $28.2^{\mathrm{ab}}$ & $10.7^{\mathrm{ab}}$ & $188.9^{\mathrm{ab}}$ & $84.61^{\mathrm{ab}}$ & $27.3^{\mathrm{b}}$ & $40.0^{\mathrm{ab}}$ & $65.1^{\mathrm{a}}$ & $2.80^{\mathrm{a}}$ & $16.0^{c}$ & $6.0^{c}$ & $12.5^{\mathrm{c}}$ & $6.1^{\mathrm{a}}$ \\
\hline Quan9311A/HB13002-50-9-7 & $88.7^{\mathrm{a}}$ & $114.9^{\mathrm{a}}$ & $27.9^{\mathrm{ab}}$ & $12.1^{\mathrm{a}}$ & $182.1^{b c}$ & $84.11^{\mathrm{ab}}$ & $27.9^{\mathrm{ab}}$ & $43.3^{\mathrm{a}}$ & $65.4^{\mathrm{a}}$ & $2.80^{\mathrm{a}}$ & $22.9^{\mathrm{b}}$ & $6.2^{\mathrm{a}}$ & $13.2^{\mathrm{b}}$ & $6.0^{\mathrm{a}}$ \\
\hline 2uans. & $87.7^{b}$ & $110.5^{b}$ & $28.5^{\mathrm{a}}$ & $11.2^{\mathrm{ab}}$ & $172.3^{c}$ & $48.39^{c}$ & $28.2^{\mathrm{a}}$ & $20.8^{c}$ & $64.1^{\mathrm{a}}$ & $2.67^{\mathrm{b}}$ & $7.5^{\mathrm{d}}$ & $5.9^{d}$ & $11.7^{d}$ & $4.8^{b}$ \\
\hline Fengliangyou4(CK) & $88.0^{\mathrm{b}}$ & $117.3^{\mathrm{a}}$ & $24.9^{c}$ & $9.6^{b}$ & $203.7^{\mathrm{a}}$ & $81.62^{b}$ & $27.5^{\mathrm{b}}$ & $35.2^{\mathrm{b}}$ & $64.5^{\mathrm{a}}$ & $2.77^{\mathrm{a}}$ & $29.2^{\mathrm{a}}$ & $6.1^{b c}$ & $14.9^{\mathrm{a}}$ & $5.9^{\mathrm{a}}$ \\
\hline
\end{tabular}

$D T H$ Number of days to heading, $P H$ plant height, $P L$ panicle length, $P N$ panicle number per plant, $N G P$ number of grains per panicle, $F E R$ fertility of the spikelet (\%), GW weight of 1000 grains, GY grains yield per plant, $H R$ head rice rate (\%), $L / W$ ratio of length to width, $C K$ chalky grain rate, $C D$ chalkiness degree, $A C$ amylose content, ASV alkali spreading value. ${ }^{a, b}, c, d$ Means followed by the same letter are not significant at the $5 \%$ significance level by the least significant difference test $(L S D=0.05)$ 


\section{Discussions}

$\mathrm{BPH}$ has been one of the important factors constraining rice yield. As the area of cultivated hybrid rice varieties gradually expands around the world, their resistance to $\mathrm{BPH}$ is attracting increasing attention by rice researchers (Hasan et al. 2015). Numerous BPH resistance genes have been introduced and pyramided into elite rice varieties, which has resulted in strongly improved capacity of rice to endure damage caused by $\mathrm{BPH}$ (Myint et al. 2012; Qiu et al. 2012; Wan et al. 2013; Suh et al. 2011). With the adaptation of $\mathrm{BPH}$ to resistant varieties and the evolution of a new biotype of BPH (Fujita et al. 2013), different $\mathrm{BPH}$ resistance genes still need to be pyramided into multiple elite hybrid rice varieties to overcome these problems. In the present study, through molecular-marker assisted backcross (MAB) breeding, we successfully transferred two BPH-resistance genes, Bph14 and Bph15, into an elite restorer line Huahui938 to obtain three improved lines, which showed enhanced resistance to $\mathrm{BPH}$ compared with the controls. When these NILs were crossed with the CMS line with the same BPH resistance genes, the hybrids (homozygous genotype with resistance loci) were resistant against BPH. Some derived hybrids had higher yield and more desirable rice qualities and agronomic performance compared with the controls. Therefore, it is possible to develop hybrid rice with high $\mathrm{BPH}$ resistance and excellent agronomic performance in the future and to solve the problem of hybrid rice being susceptible to BPH (Horgan and Crisol 2013).

The effect of BPH resistance genes would be influenced by various genetic backgrounds (Qiu et al. 2012; Jairin et al. 2010). Some previous studies demonstrated that hybrids with heterozygous Bph14 and Bph15 showed moderate resistance to $\mathrm{BPH}$ ( $\mathrm{Hu}$ et al. 2012; $\mathrm{Hu}$ et al. 2013). However, our results indicated that the heterozygote of these two genes was susceptible to $\mathrm{BPH}$ in some hybrid backgrounds at the seedling stage. In fact, $\mathrm{BPH}$ resistance genes with a partial dominant effect would show moderate resistance or would be susceptible to $\mathrm{BPH}$ in a heterozygous state under different genetic backgrounds (Liu et al. 2016). Therefore, it is essential to simultaneously stack Bph14 and Bph15 into male and female parents to guarantee hybrids with certain and durable resistance to $\mathrm{BPH}$.

With respect to rice genetic improvement, developing NILs is the optimal approach to obtain modified lines with desired traits. Molecular marker-assisted selection (MAS) is a very powerful tool to accelerate the progress of developing NILs with pyramided resistance genes and to maintain the desirable characteristics of the recurrent parent. Numerous NILs have been developed with enhanced disease resistance, such as blast resistance, sheath blight resistance, and bacterial leaf blight resistance, under different genetic backgrounds (Miah et al. 2015;
Khanna et al. 2015; Chen et al. 2014; Suh et al. 2013; Balachiranjeevi et al. 2015).

In previous studies in which NILs were developed, hundreds of RFLP or SSR markers were usually used for recovering and detecting the genetic background. It is time-consuming and low-efficiency work to identify polymorphism markers between the donor and recipient parent (Chen et al. 2000; Suh et al. 2011; Ahmed et al. 2016). However, it is easy to identify sufficient polymorphism SNP markers for background analysis due to the high-density and extensive marker positioning in an SNP array. In a comparative study estimating the similarity between the recurrent parent and the developed NILs, Khanna et al. (2015) found that the percent similarity was overestimated by SSR markers, and the background analysis using an SNP array was almost 300 times more cost effective and provided a more precise estimation due to higher resolution.

In this study, the genetic background of the improved NILs was analyzed using the SNP array RICE6K, which contains 5,102 SNP and InDel markers. We detected 550 SNP markers showing polymorphisms between Huahui938 and B5 in an efficient amount of time. Jiang et al. (2015) examined the genetic background recovery of developed lines using the same RICE6K array and obtained accurate results. Mi et al. (2016) obtained a modified 9311 line carrying stacked genes $55-n$ and $S 5-n$ with an RPG recovery rate higher than $99 \%$ using RICE6K, and a higher density SNP array in the $\mathrm{BC}_{5}$ generation and the agronomic performance of those NILs did not differ from the recurrent parent 9311. Thus, as the cost of genotyping using an SNP array decreases, it will be possible to use this powerful tool to develop NILs in all breeding schemes in the near future.

In the present study, the hybrid combination Luohong4A/HB13002-50-9-7 exhibited BPH resistance, high yield and good rice quality similar to that of Fengliangyou4. We affirm that this hybrid can be used for rice production in the $\mathrm{BPH}$ epidemic region in southern China. The yield of Quan9311A/HB13002-50-9-7 was the highest among the tested combinations even though it showed no resistance against $\mathrm{BPH}$ at the seedling stage. We believe this can be used as a high yield hybrid for production in areas where BPH does not occur.

\section{Conclusions}

In this study, we successfully transferred both Bph14 and Bph15 into the CMS rice restorer line, and three BPH-resistance NILs were obtained. The results showed that when both male and female parents contained the same $\mathrm{BPH}$ resistance genes, the hybrids were resistant against BPH. The combination Luohong4A/HB1300250-9-7 obtained in this study had high yield, BPH resistance, superior rice quality and desirable agronomic 
performance, showing a remarkable commercial potential for hybrid rice production in $\mathrm{BPH}$ epidemic areas.

\section{Methods}

\section{Rice Materials and Breeding Scheme}

Huahui938, an elite indica CMS rice restorer line with good combined abilities of yield, good rice quality and high blast resistance but susceptible to $\mathrm{BPH}$, was used as the recurrent parent. B5, a highly resistant line whose resistance genes Bph14 and Bph15 were obtained from wild rice (Oryza officinalis), was used as the donor parent (Huang et al. 2001). Taichung Native 1 (TN1), a line highly susceptible to $\mathrm{BPH}$, was used as the negative control to evaluate BPH resistance. Two CMS lines, Quan9311A and Luohong4A, were used as the maternal parents to produce the hybrid rice. Quan9311A was developed by the Quanyin Seed Company, Hefei, China, and has good combined abilities of yield and high rice quality but is susceptible to BPH. Luohong4A, which was developed by Wuhan University, Wuhan, China, harbors homozygous Bph14 and Bph15 loci and is resistant to $\mathrm{BPH}$ (Zhu et al. 2013). In addition, Fengliangyou4, which was developed by the Fengle Seed Company, Anhui province, China, and is an existing check variety for the registration of new rice varieties in China, was used as the control for BPH resistance and agronomic performance evaluation of hybrid rice in this study. The overall breeding scheme consisted of a recurrent backcrossing procedure including one crossing, three generations of backcrossing and four generations of self-fertilization, combined with MAS in each generation (Fig. 4). A cross was made between the recurrent parent Huahui938 and the donor parent $\mathrm{B} 5$. In backcrossing generations from $\mathrm{BC}_{1} \mathrm{~F}_{1}$ to $\mathrm{BC}_{3} \mathrm{~F}_{1}$, selected individuals with a phenotype similar to Huahui938 and heterozygous Bph14 and Bph15

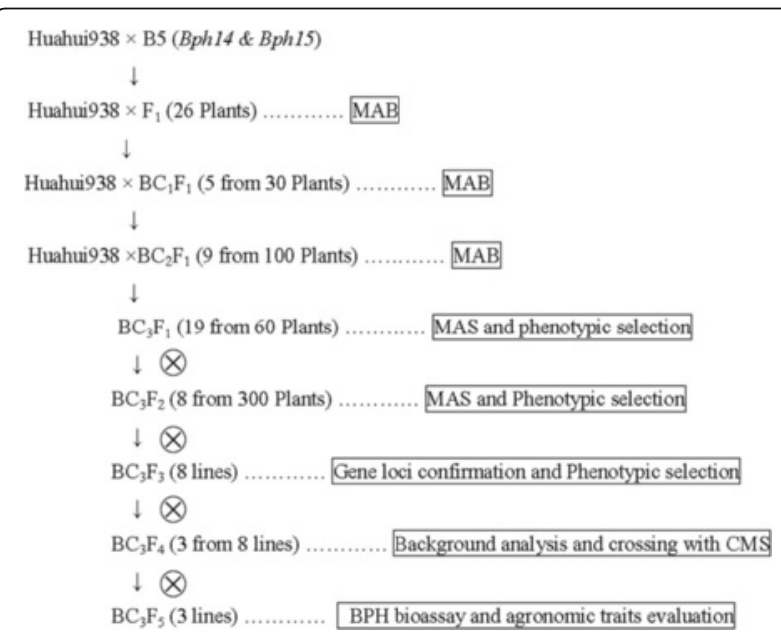

Fig. 4 Procedure for the development of NILs with homozygous Bph14 and Bph15 loci in the genetic background of Huahui938 loci were backcrossed to Huahui938. Three near-isogenic lines (NILs) bearing both homozygous Bph14 and Bph15 loci were generated from the $\mathrm{BC}_{3} \mathrm{~F}_{2}$ segregation population. Finally, the RICE6K array was used to verify the background of the improved lines.

\section{DNA Extraction, PCR, Markers and Genotyping}

Total genomic DNA was extracted from fresh rice leaves using the modified CTAB method following Dellaporta et al. (1983). PCR reactions were carried out as described by Jiang et al. (2015). PCR reactions were performed using

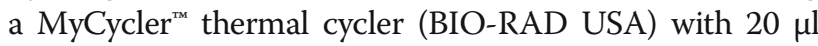
reaction mixture that contained $20 \mathrm{ng}$ genomic DNA, $10 \mathrm{mM}$ Tris- $\mathrm{HCl}$ (pH 9.0), $50 \mathrm{mM} \mathrm{KCl}, 2.5 \mathrm{mM} \mathrm{MgCl}_{2}$, $2 \mathrm{mM}$ dNTPs, $10 \mu \mathrm{M}$ each of the forward and reverse primers, and 1 unit of Taq DNA polymerase. The PCR amplification program consisted of one cycle of denaturation at $95{ }^{\circ} \mathrm{C}$ for $5 \mathrm{~min}$, followed by 35 cycles at $95^{\circ} \mathrm{C}$ for $30 \mathrm{~s}, 56{ }^{\circ} \mathrm{C}$ for $30 \mathrm{~s}, 72{ }^{\circ} \mathrm{C}$ for $45 \mathrm{~s}$, with a final extension at $72{ }^{\circ} \mathrm{C}$ for $7 \mathrm{~min}$. The InDel marker $76-2$ (primer sequences F: 5'-GCACATACAGAAATGGTGAA-3', R: 5'-GGCAAGGGACATGTAGTAAC-3') linked to Bph14 was used to select positive individual plants with respect to the Bph14 locus in each generation ( $\mathrm{Du}$ et al. 2009). The SSR marker MS5 (primer sequences F: 5' - TTGT GGGTCCTCATCTCCTC-3', R: 5' -TGACAACTTGTGC AAGATCAAA-3') linked to Bph15 (Yang et al. 2004) and the InDel marker InD4 (primer sequences F: 5'-AGAAT GCTAAAGATGACTGAA-3', R: 5'-AACGGTATTGTTC TTGTCTAA-3'), which was more tightly linked to Bph15 ( $\mathrm{Lv}$ et al. 2014), were used to select individual positive plants in each generation. The PCR products were analyzed using $4 \%$ denaturing polyacrylamide gel electrophoresis. In addition, the whole-genome single nucleotide polymorphism (SNP) array RICE6K was used to analyze the genetic background similarity of the selected NILs with respect to the recurrent parent Huahui938. This SNP array was developed based on Infinium technology, which contains 5102 SNP and insertion-deletion (InDel) markers evenly distributed on the 12 chromosomes of rice with an average density of $12 \mathrm{SNPs}$ per $1 \mathrm{Mb}$ (Yu et al. 2014). For each improved line, total DNA was extracted from 20 plants leaves. Genetic background similarity analysis was performed at the Life Science and Technology Center, China National Seed Group Co., LTD (Wuhan, China), according to Infinium HD Assay Ultra Protocol (http:// www.illumina.com/).

\section{BPH Bioassay for the Selected NILs and Their Hybrids}

The BPH resistance evaluations of the selected NILs and their hybrids were conducted in a net house. Huahui938, Quan9311A, Fengliangyou4 and TN1 were used as susceptible controls, while Luohong4A and the donor parent B5 were used as the $\mathrm{BPH}$ resistant controls. 
The BPH samples used in the test were collected from the field and were continuously reared on TN1 in the laboratory at Huazhong Agricultural University (HZAU), Wuhan, China. The bioassay was performed using a modified seedling bulk test following the SSST (Velusamy et al. 1986; Horgan 2009). Thirty pre-germinated seeds of each entry, including the improved lines and controls with three replications, were evenly sown in $7 \times 7 \times 8 \mathrm{~cm}$ plastic plates. All entries were randomly arranged in a large pool. When the seedlings reached the second leaf stage, they were thinned to 20 plants per plate. At the third-leaf stage, the seedlings were infested with 2nd-3rd-instar $\mathrm{BPH}$ nymphs at a rate of $8-10$ insects per seedling, and the water was maintained at a depth of approximately $0.5 \mathrm{~cm}$ above the root until the evaluation was completed. All seedlings were patted once, $24 \mathrm{~h}$ after the infestation, to evenly redistribute the nymphs. When all TN1 plants died, the other plants were examined and the following scores were given: 0 (no damage), 1 (very slight damage), 3 (the first and second leaf of most plants were yellowing), 5 (yellowing, nearly half of the plants had wilted or were dead), 7 (more than half of the plants were dead and the rest were seriously dwarfed) and 9 (all plants were dead) according to the modified criteria based on the Standard Evaluation System for Rice (IRRI 2002). An average resistance score of 0.1-1.9 was designated as highly resistant (HR), $2.0-3.9$ as resistant (R), 4.0-5.9 as moderately resistant (MR), 6.0-7.9 as susceptible (S), and 8.0-9.0 as highly susceptible (HS) (IRRI 2002).

\section{Evaluation of Agronomic and Grain Quality Traits}

To evaluate the agronomic and rice quality traits, Huahui938, B5, three improved NILs and their derived hybrids from Quan9311A and Luohong4A were planted in a randomized complete block design with three replications at Wuhan, China, in the summer of 2015 under natural field conditions. Each plot consisted of three rows with 10 plants per row at a planting density of $16 \mathrm{~cm}$ between plants within a row and $20 \mathrm{~cm}$ between rows. The agronomic and rice quality traits were measured according to the standard evaluation system for rice (IRRI 2002). Five mature plants in the middle row were harvested for measurements. The agronomic traits evaluated included number of days to heading $(\mathrm{DTH})$, plant height $(\mathrm{PH})$, panicle length $(\mathrm{PL})$, panicle number (PN), number of grains per panicle (NGP), fertility of the spikelet (FER), grain yield (GY), and 1000-grain weight $(\mathrm{GW})$. The harvested seeds were stored at room temperature for three months at $14 \%$ moisture content. The following grain quality traits were tested: head rice rate $(\mathrm{HR})$, ratio of grain length to width $(\mathrm{L} / \mathrm{W})$, chalky grain rate $(\mathrm{CK})$, chalkiness degree $(\mathrm{CD})$, amylose content (AC) and alkali spreading value (ASV).

\section{Data analysis}

The background recovery percentage $(\mathrm{BRP})$ was calculated using the following formula: BRP $(\%)=(R+1 / 2 H) \times 100 / P$. In this formula, $\mathrm{R}, \mathrm{H}$, and $\mathrm{P}$ indicate the number of SNP markers homozygous for Huahui938, the number of markers that remained heterozygous, and the total number of polymorphic markers between Huahui938 and B5, respectively. One-way ANVOA and least significant difference (LSD) tests were performed using the SAS statistical software package (version 9.0; SAS Institute, Cary, NC).

\section{Abbreviations \\ AC: Amylose content; ASV: Alkali spreading value; BPH: Brown planthopper; BRP: Background recovery percentage; CD: Chalkiness degree; CK: Chalky grain rate; CMS: Cytoplasmic male sterility; CTAB: Hexadecyl trimethyl ammonium bromide; DTH: Number of days to heading; FER: Fertility of the spikelet; GW: Grain weight; GY: Grain yield; HR: Head rice rate; \\ Indel: Insertion-deletion; IRRI: International Rice Research Institute; L/W: Ratio of grain length to width; LSD: Least significant difference tests; \\ MAB: Molecular marker-assisted backcross; MAS: Marker-assisted selection; NGP: Number of grains per panicle; NILs: Near-isogenic lines; \\ PCR: Polymerase chain reaction; PH: Plant height; PL: Panicle length; PN: Panicle number; RGSV: Rice grassy stunt virus; RPG: Recurrent parent genome; RRSV: Rice ragged stunt virus; RWSV: Rice wilted stunt virus; SES: Standard evaluation system; SNP: Single nucleotide polymorphism; SSR: Simple sequence repeat; SSST: Standard Seedbox Screening Test; TGMS: Thermo-sensitive genetic male-sterile lines}

\section{Acknowledgments}

This research was partially supported by the National High Technology Research and Development Programs of China (863 Programs, "Breeding of New Varieties of Green Super Rice", Grant No. 2014AA10A604), Wuhan municipal government R \& D programs (Grant No. 2014020202010136) and the Bill \& Melinda Gates Foundation "Green Super Rice for the Resources-Poor of Africa and Asia".

\section{Authors' Contributions}

HBW carried out the experiments, partial MAS, bioassay of BPH resistance and evaluation of agronomic and rice grain quality traits. STY did partial MAS. TMM conceptualized the study and did phenotype selection. HBW and TMM prepared manuscript together. All authors read and approved the final manuscript.

\section{Competing Interests}

The authors declare that they have no competing interests.

Received: 8 March 2016 Accepted: 24 September 2016

Published online: 04 October 2016

\section{References}

Ahmed F, Rafii MY, Imail MR, Juraimi AS, Rahim HA, Tanweer FA, Latif MA (2016) Recurrent parent genome recovery in different populations with the introgression of Sub1 gene from a cross between MR219 and Swarna-Sub1. Euphytica 207:605-618

Balachiranjeevi CH, Bhaskar NS, Abhilash V. Akanksha S, Viraktamath BC, Madhav MS, Hariprasad AS, Laha GS, Prsad MS, Balachandran SM (2015) Markerassisted introgression of bacterial blight and blast resistance into DRR17B, an elite, fine-grain type maintainer line of rice. Mol Breeding 35:151

Cai Z, Li J, Zhou D, Fu H (2015) Improving the resistance of japonica in the north of Zhejiang province by marker-assisted selection (in Chinese with English abstract). Mod Agric Sci Technol 22:117-122

Chen S, Lin XH, Xu CG, Zhang Q (2000) Improvement of bacterial blight resistance of 'Minghui 63', an elite restorer line of hybrid rice, by molecular marker-assisted selection. Crop Sci 40:239-244

Chen ZX, Zhang YF, Feng F, Feng MH, Jiang W, Ma YY, Pan CH, Hua HL, Li GS, Pan XB, Zuo SM (2014) Improvement of japonica rice resistance to sheath blight by pyramiding qSB-9 ${ }^{\text {TQ }}$ and qSB-7 ${ }^{\text {TQ }}$. Field Crop Res 161:118-227 
Dellaporta SL, Wood J, Hicks JB (1983) A plant DNA minipreparation: version II. Plant Mol Biol Rep 1:19-21

Du B, Zhang W, Liu B, Hu J, Wei Z, Shi Z, He R, Zhu L, Chen R, Han B, He G (2009) Identification and characterization of Bph14, a gene conferring resistance to brown planthopper in rice. Proc Natl Acad Sci U S A 106:22163-22168

Fujita D, Kohli A, Horgan FG (2013) Rice resistance to planthoppers and leafhoppers. Criti Rev Plant Sci 32:162-191

Hasan MM, Rafii MY, Ismail MR, Mahmood M, Rahim HA, Alam MA, Ashkani S, Malek MA, Latif MA (2015) Marker-assisted backcrossing: a useful method for rice improvement. Biotechnol Biotechnol Equip 29:237-254

Horgan F (2009) Mechanisms of resistance: A major gap in understanding planthopper-rice interactions. In: Heong KL, Hardy B (eds) Planthoppers: New Threats to the Sustainability of Intensive Rice Production Systems in Asia. International Rice Research Institute, Los Baños, pp 281-302

Horgan FG, Crisol E (2013) Hybrid rice and insect herbivores in Asia. Entomol Exp Appl 148:1-19

Hu J, Li X, Wu C, Yang C, Hua H, Gao G, Xiao J, He Y (2012) Pyramiding and evaluation of the brown planthopper resistance genes Bph14 and Bph15 in hybrid rice. Mol Breed 29:61-69

Hu J, Cheng M, Gao G, Zhang Q, Xiao J, He Y (2013) Pyramiding and evaluation of three dominant brown planthopper resistance genes in the elite indica rice 9311 and its hybrids. Pest Manag Sci 69:802-808

Hu W, Li Y, Hu K, Jiang Y, Zhang Y (2015) Improvement BPH-resistance of rice culitivar Guinongzhan by marker-assisted selection for BPH-resistant genes (in Chinese with English abstract). Mol Plant breeding 13:951-960

Huang Z, He G, Shu L, Li X, Zhang Q (2001) Identification and mapping of two brown planthopper resistance genes in rice. Theor Appl Genet 102:929-934

IRRI (2002) Standard evaluation system for rice (SES). International Rice Research Institute, Los Baños

Jairin J, Sansen K, Wongboon W, Kothcharerk J (2010) Detection of a brown planthopper resistance gene bph4 at the same chromosomal position of Bph3 using two different genetic backgrounds of rice. Breed Sci 60:71-75

Jiang J, Mou T, Yu H, Zhou F (2015) Molecular breeding of thermo-sensitive genic male sterile (TGMS) lines of rice for blast resistance using Pi2 gene. Rice 8:11

Khanna A, Sharma V, Ellur RK, Shikari AB, Krishnan SG, Singh UD, Prakash G, Sharma TR, Rathour R, Variar M, Prashanthi SK, Nagarajan M, Vinod KK, Bhowmick PK, Singh NK, Prabhu KV, Singh BD, Singh AK (2015) Development and evaluation of near-isogenic lines for major blast resistance gene(s) in Basmati rice. Theor Appl Genet 128:1243-1259

Li J, Xia M, Qi H, He G, Wan B, Zha Z (2006) Marker-assisted selection for brown planthopper (Nilaparvata lugens Stål) resistance genes Bph14 and Bph15 in rice. Science Agricultura Sinica 39:2132-2137

Li J, Chen Q, Wang L, Liu J, Shang K, Hua H (2011) Biological effects of rice harbouring Bph14 and Bph15 on brown planthopper, Nilaparvata lugens. Pest Manag Sci 67:528-534

Liu Y, Chen L, Liu Y, Dai H, He J, Kang H, Pan G, Huang J, Qiu Z, Wang Q, Hu J, Liu L, Chen Y, CHeng X, Jiang L, Wan J (2016) Marker assisted pyramding of two brown planthopper resistance genes, Bph3 and Bph27(t), into elite rice cultivars. Rice 9:27

Lv W, Du B, Shangguan X, Zhao Y, Pan Y, Zhu L, He Y, He G (2014) BAC and RNA sequencing reveal the brown planthopper resistance gene BPH15 in a recombination cold spot that mediates a unique defense mechanism. BMC Genomics 15:674

Mi J, Li G, Huang J, Yu H, Zhou F, Zhang Q, Ouyang Y, Mou T (2016) Stacking $55-n$ and $f 5-n$ to overcome sterility in indica-japonica hybrid rice. Theor Appl Genet 129:563-575

Miah G, Rafii MY, Ismail MR, Puteh AB, Rahim HA, Latif MA (2015) Recurrent parent genome recovery analysis in a marker-assisted backcrossing program of rice (Oryza stiva L.) C.R. Biologies 338:83-94

Myint KK, Fujita D, Matsumura M, Sonoda T, Yoshimura A, Yasui H (2012) Mapping and pyramiding of two major genes for resistance to the brown planthopper (Nilaparvata lugens [Stål]) in the rice cultivar ADR52. Theor Appl Genet 124:495-504

Qiu Y, Guo J, Jing S, Zhu L, He G (2012) Development and characterization of japonica rice lines carrying the brown planthopper-resistance genes BPH12 and BPH6. Theor Appl Genet 124:485-494

Seck PA, Diagne A, Mohanty S, Wopereis MCS (2012) Crops that feed the world 7: Rice. Food Sec 4:7-24

Suh JP, Yang SJ, Jeung JU, Pamplona A, Kim JJ, Lee JH, Hong HC, Yang Cl, Kim YG, Jena KK (2011) Development of elite breeding lines conferring Bph18 gene-derived resistance to brown planthopper (BPH) by marker-assisted selection and genome-wide background analysis in japonica rice (Oryza sativa L.). Field Crop Res 120:215-222

Suh JP, Jeung JU, Noh TH, Cho YC, Park SH, Park HS, Shin MS, Kim CK, Jena KK (2013) Development of breeding lines with three pyramided resistance genes that confer broad- spectrum bacterial blight resistance and their molecular analysis in rice. Rice 6:5

Sun L, Su C, Wang C, Zhai H, Wan J (2005) Mapping of a major resistance gene to the Brown Plant hopper in the rice cultivar Rathu Heenati. Breed Sci 55:391-396

Tanksley SD, Young ND, Paterson AH, Bonierbale MW (1989) RFLP mapping in plant breeding: new tools for old science. Biotechnology 7:257-264

Velusamy R, Heinrichs EA, Medrano FG (1986) Greenhouse techniques to identify field resistance to the brown planthopper, Nilaparvata lugens (Stål) (Homoptera: Delphacidea), in rice cultivars. Crop Prot 5:328-333

Wan B, Zha Z, Li J, Xia M, Du X, Lin Y, Yin D (2013) Development of elite rice restorer lines in the genetic background of R022 possessing tolerance to brown planthopper, stem borer, leaf folder and herbicide through marker-assisted breeding. Euphytica 195:129-142

Wang Y, Cao L, Zhang Y, Cao C, Liu F, Huang F, Qiu Y, Li R, Lou X (2015) Map-based cloning and characterization of BPH29, a B3 domain-containing recessive gene conferring brown planthopper resistance in rice. J Exp Bot 66:6035-6045

Xia M, Wan B, Li J, Zha Z, Du X, Qi H (2010) Breeding and application of new quality medimun indica hybrid rice (in Chinese with English abstract). Hybrid Rice 25:18-20

Yang H, You A, Yang Z, Zhang F, He R, Zhu L, He G (2004) High-resolution genetic mapping at the Bph15 locus for brown planthopper resistance in rice (Oryza sativa L.). Theor Appl Genet 110:182-191

Yu H, Xie W, Li J, Zhou F, Zhang Q (2014) A whole-genome SNP array (RICE6K) for genomic breeding in rice. Plant Biotechnol J 12:28-37

Zhu R, Huang W, Hu J, Liu W, Zhu Y (2013a) Breeding of new sterile line Luohong 4A of Honglian type hybrid rice (in Chinese with English abstract). J Wuhan Univ ( Nat Sci Ed ) 59:33-36

Zhu R, Huang W, Hu J, Liu W, Zhu Y (2013b) Breeding and utilization of hybrid rice Liangyou234 and NMS line Bph68S resistance to brown planthopper (in Chinese with English abstract). J Wuhan Univ ( Nat Sci Ed ) 59:24-28

\section{Submit your manuscript to a SpringerOpen ${ }^{\circ}$ journal and benefit from:}

- Convenient online submission

- Rigorous peer review

- Immediate publication on acceptance

- Open access: articles freely available online

- High visibility within the field

- Retaining the copyright to your article

Submit your next manuscript at springeropen.com 